\title{
Electrochemical Fabrication of Nanostructures on Porous Silicon for Biochemical Sensing Platforms
}

\author{
Euna Ko, Joonki Hwang, Ji Hye Kim, Joo Heon Lee, Sung Hwan Lee, Van-Khue Tran, \\ Woo Sung Chung, Chan Ho Park, Jaebum Choo, and Gi Hun Seong ${ }^{\dagger}$
}

Department of Bionano Engineering, Hanyang University, Ansan, 425-791, South Korea

\begin{abstract}
We present a method for the electrochemical patterning of gold nanoparticles (AuNPs) or silver nanoparticles (AgNPs) on porous silicon, and explore their applications in: (1) the quantitative analysis of hydroxylamine as a chemical sensing electrode and (2) as a highly sensitive surface-enhanced Raman spectroscopy (SERS) substrate for Rhodamine 6G. For hydroxylamine detection, AuNPs-porous silicon can enhance the electrochemical oxidation of hydroxylamine. The current changed linearly for concentrations ranging from $100 \mu \mathrm{M}$ to $1.32 \mathrm{mM}\left(R^{2}=0.995\right)$, and the detection limit was determined to be as low as $55 \mu \mathrm{M}$. When used as SERS substrates, these materials also showed that nanoparticles decorated on porous silicon substrates have more SERS hot spots than those decorated on crystalline silicon substrates, resulting in a larger SERS signal. Moreover, AgNPs-porous silicon provided five-times higher signal compared to AuNPs-porous silicon. From these results, we expect that nanoparticles decorated on porous silicon substrates can be used in various types of biochemical sensing platforms.
\end{abstract}

Keywords Porous silicon, electrochemical deposition, nanoparticles, electrochemical sensor, SERS substrate

(Received February 1, 2016; Accepted February 19, 2016; Published June 10, 2016)

\section{Introduction}

Silicon is one of the most powerful semiconducting materials because it is robust and has high thermal conductivity, can be easily doped, and is low in cost. Nowadays, silicon nanomaterials, such as nanodots, nanowires, and porous silicon, have drawn great interest due to their favorable biocompatibility, large surface area, surface tailorability, improved multifunctionality, low cost, and their compatibility with conventional silicon technologies. ${ }^{1-3}$ Porous silicon is especially attractive for various applications, including electronics, actuators, and sensors, because it is considered to be more efficient and selective than crystalline silicon due to its high surface area and high surface energy.,5 However, freshly prepared porous silicon is hydrophobic and unstable because the $\mathrm{Si}-\mathrm{H}_{\mathrm{x}}$ groups of the internal surface of porous silicon are very reactive. Metastable $\mathrm{Si}-\mathrm{H}_{\mathrm{x}}$ bonds can undergo spontaneous oxidation under ambient conditions, which can degrade the surface structures. ${ }^{6}$ Therefore, for applications in biochemical sensing platforms, it is important to stabilize the surface of porous silicon.

The general method used to stabilize porous silicon surfaces is to modify the $\mathrm{Si}-\mathrm{O}$ bonds or $\mathrm{Si}-\mathrm{C}$ bonds on the porous silicon surface. ${ }^{7}$ Another way to passivate the surface is via the electrochemical deposition of noble metals (e.g., Au, Ag, and $\mathrm{Cu}$ ), which is an alternative method to create a stable interface between the electrolyte and porous silicon. ${ }^{8}$ Noble-metal nanoparticles have been explored as supporting materials to

† To whom correspondence should be addressed.

E-mail: ghseong@hanyang.ac.kr modify the surface and to enhance the catalytic activity of the substrate. These are appealing because of their controllable nanoparticle structure, efficient optical properties, strong stability, limited aggregation, and high electrochemical performance..$^{910}$ Here, gold nanoparticles (AuNPs) or silver nanoparticles (AgNPs) were decorated onto porous silicon by electrochemical deposition; these materials were then utilized as biochemical sensing platforms for electrochemical hydroxylamine detection and as substrates for surface-enhanced Raman scattering (SERS).

Hydroxylamine plays an important role in the preparation of polymers as a reducing agent, and takes part in many biological processes as an intermediate. Therefore, rapid, reproducible, and highly sensitive detection of hydroxylamine is required in a variety of areas (e.g., industrial, environmental, clinical diagnostics, and biological processing). Compared to other methods of hydroxylamine detection, the electrochemical determination of hydroxylamine has a variety of advantages because electrochemical analysis is portable, inexpensive, and fast; it also has a sensitive detection method with low detection limits. ${ }^{11}$ In addition, electrochemical detection with nanoparticles as redox-active materials offers significant advantages in the development of electrochemical sensors. Nanoparticledeposited electrodes are attractive sensors for the electrochemical detection of hydroxylamine due to their high active surface area, efficient electrochemical properties, good stability in aqueous solutions, and high sensitivity. ${ }^{12}$

SERS is one of the most widely researched analytical tools due to its ability to detect enhanced Raman signals for molecules adsorbed on special metallic surfaces with nanoscale roughness. Commonly used SERS substrates are aggregated nanoparticle colloids or roughened metal electrodes. Rough metal substrates 
are stable and more reproducible than colloidal solutions, but they are not as sensitive and can be difficult to fabricate. ${ }^{13}$ However, nanoparticles on porous silicon have become promising materials as sensitive SERS metal substrates that can be prepared easily. ${ }^{14}$ The high surface area resulting from the roughness of porous silicon increases the number of molecules that produce the highly enhanced SERS signal. Structured AuNPs or AgNPs exhibit the largest enhancements with visible/near-infrared excitation, and have demonstrated an enhanced Raman-scattering efficiency. ${ }^{15}$

In this study, we report the electrochemical patterning of nanoparticles on porous silicon and their applications in: 1) chemical sensors for hydroxylamine detection and 2) the development of highly sensitive SERS substrates. Porous silicon was prepared by an electrochemical etching process of crystalline silicon wafers with illumination, and nanoparticles were deposited on the porous silicon using an electrochemical method. For hydroxylamine detection, AuNPs were decorated on porous silicon; this enhanced the electrochemical oxidation of hydroxylamine, resulting in highly sensitive sensors with rapid response. In another application, AgNPs were deposited on porous silicon, and the SERS activity was investigated by measuring the Raman signal of Rhodamine $6 \mathrm{G}$ absorbed onto the as-prepared substrates. The SERS spectra of AgNPs on porous silicon emitted higher Raman signals for Rhodamine 6G compared to the SERS spectra emitted from crystalline silicon. This is caused by the rough surface of the porous silicon and the large enhancement factor of the metal nanoparticles.

\section{Experimental}

\section{Materials}

N-type, (100)-oriented, 4-inch silicon wafers doped with phosphorus (resistivity: $1-10 \Omega \mathrm{cm}^{-1}, 520 \pm 5 \mu \mathrm{m}$ thick) were purchased from Sehyoung Wafertech (Seoul, South Korea). Sulfuric acid, hydroxylamine, hydrofluoric acid (HF), hydrochloric acid, Rhodamine 6G, and gold(III) chloride $\left(\mathrm{HAuCl}_{4}\right)$ were obtained from Sigma-Aldrich (St. Louis, MO, USA). Sodium chloride and silver nitrate $\left(\mathrm{AgNO}_{3}\right)$ were purchased from Junsei Chemical Co. (Tokyo, Japan). Ethanol and acetone were obtained from Samchun Pure Chemical Co. (Seoul, South Korea). Phosphate-buffered saline (PBS, pH 7.2) was obtained from Biosesang, Inc. (Sungnam, South Korea). The positive photoresist polymer (PR, AZ4620) was purchased from Clariant Corporation (Somerville, NJ, USA). A halogen lamp (64401 ECO $100 \mathrm{~W}$ ) was obtained from Osram (Munich, Germany). All other chemicals were of analytical grade, and the aqueous solutions were prepared with deionized (DI) water with a resistivity of $18.2 \mathrm{M} \Omega \mathrm{cm}^{-1}$.

\section{Fabrication of porous silicon}

The silicon wafers were cut into square pieces $(1 \times 1 \mathrm{~cm})$ and pre-cleaned by immersion in $1 \mathrm{wt} \% \mathrm{HF}$ for $5 \mathrm{~min}$, ethanol for $10 \mathrm{~min}$, and acetone for $10 \mathrm{~min}$. The samples were washed with DI water and dried under a stream of nitrogen gas. A standard photolithography process was then employed to generate a template of the photoresist polymer (AZ4620) onto the clean n-type crystalline silicon surface. The patterned silicon wafer was etched in a mixture of HF, ethanol, and DI water at a volumetric ratio of 1:1:2. A constant potential of $4 \mathrm{~V}$ was applied for 15 min under backside illumination using a $100-\mathrm{W}$ halogen lamp at a distance of $10 \mathrm{~cm}$. After anodization, the samples were washed with DI water and dried under a stream of nitrogen gas.

\section{Electrochemical deposition of nanoparticles}

AuNPs were electrochemically deposited on porous silicon pre-patterned with the photoresist polymer. Porous silicon, which was used as the working electrode, was dipped in a $9 \mathrm{mM}$ $\mathrm{HAuCl}_{4}$ solution at potential range from -1.0 to $0.6 \mathrm{~V}$ (vs. $\mathrm{Ag} / \mathrm{AgCl}$ ) using the cyclic voltammetry (CV) technique. The electrochemical deposition of AgNPs on porous silicon was performed with a $\mathrm{CV}$ technique in a $9 \mathrm{mM} \mathrm{AgNO}_{3}$ solution at an applied potential range from -1.0 to $0.6 \mathrm{~V}$ (vs. $\mathrm{Ag} / \mathrm{AgCl}$ ). CVs were recorded for five cycles at a scan rate $20 \mathrm{mV} \mathrm{s}^{-1}$. Afterward, the nanoparticle-deposited porous silicon was washed with DI water and dried under a mild stream of nitrogen gas. The area of each working electrode was $0.0625 \mathrm{~cm}^{2}$.

\section{Electrochemical analysis of hydroxylamine}

Electrochemical measurements were carried out with a CHI660C electrochemical analyzer ( $\mathrm{CH}$ Instruments, Inc., USA). A three-electrode configuration was employed, in which the AuNPs-decorated porous silicon electrode was used as the working electrode and placed into a cell. A clean platinum wire $(\mathrm{d}=0.3 \mathrm{~mm}$ ) and $\mathrm{Ag} / \mathrm{AgCl}$ (saturated in $3 \mathrm{M} \mathrm{KCl}$ ) were used as counter and reference electrodes, respectively. All cyclic voltammetry measurements were performed in a $0.1 \mathrm{M}$ PBS buffer ( $\mathrm{pH}$ 7.2) solution as the electrolyte with a scan rate of $50 \mathrm{mVs}^{-1}$.

\section{Preparation of SERS substrate}

Rhodamine 6G (R6G) solutions $(10 \mu \mathrm{L})$ with different concentrations were dropped onto the AgNPs-deposited porous silicon and allowed to dry for $1 \mathrm{~h}$ at room temperature. SERS measurements were performed using an inVia Raman microscope system (Renishaw, UK). A He-Ne laser, operating at $632.8 \mathrm{~nm}$, was used as the excitation source with a laser power of approximately $50 \mathrm{~mW}$. The Rayleigh line was removed from the collected Raman scattering using a holographic notch filter located in the collection path. Raman scattering was collected using a charge-coupled device (CCD) camera at a spectral resolution of $1 \mathrm{~cm}^{-1}$. An additional CCD camera was fitted to an optical microscope to obtain optical images. A $20 \times$ objective lens was used to focus the laser spot on the substrate. All of the Raman spectra reported here were collected for an exposure time of $0.1 \mathrm{~s}$ in the range of $600-$ $1700 \mathrm{~cm}^{-1}$

\section{Results and Discussion}

Porous silicon substrates were fabricated by electrochemical etching of n-type crystalline silicon wafers in an aqueous HF solution with backside illumination (Fig. 1). The pore morphology, porosity, and porous layer thickness were controlled by changing the electrochemical anodization parameters, such as the current density, HF concentration, and etching time. ${ }^{16}$ The morphology of porous silicon was examined with FE-SEM after anodization at a constant voltage of $4 \mathrm{~V}$ for an etching time of $15 \mathrm{~min}$ (Fig. 2). The obtained porous silicon consisted of unidirectionally-aligned pores running perpendicular to the surface, where the average pore diameter and thickness of the porous layer were about 2 and $10 \mu \mathrm{m}$, respectively. The formation mechanism of the porous silicon has been proposed by the following net reaction: ${ }^{17}$

$$
\mathrm{Si}+6 \mathrm{HF}+\mathrm{h}^{+} \longrightarrow \mathrm{SiF}_{6}^{2-}+\mathrm{H}_{2}+4 \mathrm{H}^{+}+\mathrm{e}^{-} .
$$

For the dissolution of silicon during the anodization process, 


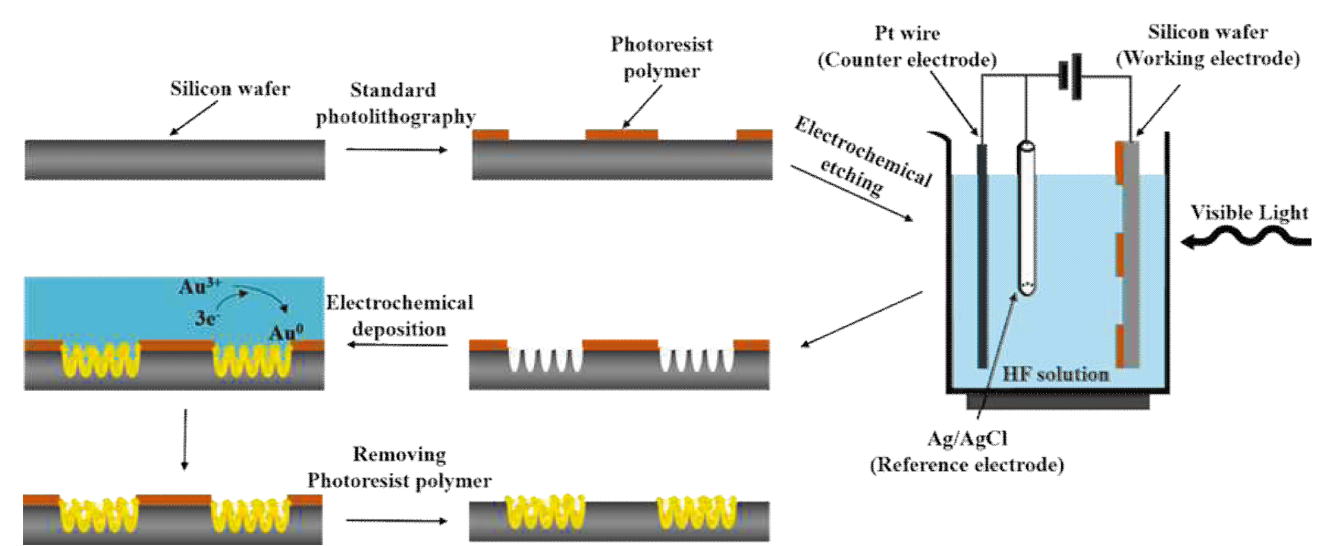

Fig. 1 Schematic of the fabrication process for porous silicon and gold nanoparticles.

an external source of holes $\left(\mathrm{h}^{+}\right)$is necessary, because n-type silicon has a low hole density. To provide an external hole source, backside illumination was used to generate holes in the n-type silicon. ${ }^{18}$ During illumination, the generated holes diffused to the front side of the silicon substrate and reacted with the negative solvated $\mathrm{F}^{-}$ions at the silicon/electrolyte interface, resulting in the dissolution of the silicon surface. ${ }^{16}$ The etching rate is determined by hole accumulation in the regions adjacent to the $\mathrm{HF}$ electrolyte and silicon atoms. The hydrogen bubbles formed during the etching process were removed by ethanol, thereby preventing these bubbles from sticking to the porous silicon layer.

To apply the fabricated porous silicon substrates to biochemical sensing platforms, AuNPs or AgNPs were decorated onto the substrates by electrochemical deposition (Fig. 3). Freshly fabricated porous silicon is metastable and reactive due to the hydrogen-terminated $\left(\mathrm{Si}-\mathrm{H}_{\mathrm{x}}\right)$ porous silicon surface. ${ }^{19}$ Therefore, it is necessary to coat the porous $\mathrm{Si}$ surface in order to improve its stability. For passivation of the porous surface, metal nanoparticles were deposited by an electrochemical method. By immersing the porous silicon substrates into $\mathrm{Au}$ or $\mathrm{Ag}$ ion solutions for $1 \mathrm{~min}$ with stirring, the pores were filled with the solution, which contained metal ions; this led to immediate electroless deposition due to simultaneous silicon oxidation and metal reduction. As a result, large numbers of $\mathrm{Au}$ or $\mathrm{Ag}$ nuclei were generated on the porous silicon surface. After dipping the porous silicon into the metal ion electrolyte for $1 \mathrm{~min}$, electrochemical deposition was performed to fabricate AuNPs or AgNPs on the porous silicon substrates. The metal nanoparticles can be grown easily because of the presence of metal nuclei. As shown in Fig. 4, the standard equilibrium potentials of $\mathrm{AuCl}_{4}-\mathrm{Au}$ and $\mathrm{Ag}^{+} / \mathrm{Ag}$ are 0.76 and $0.8 \mathrm{~V}$ (vs. $\mathrm{Ag} / \mathrm{AgCl})$, respectively. To control the nanoparticle size and homogeneity, a lower potential range $(-1.0$ to $+0.6 \mathrm{~V})$ than the standard equilibrium potential of metal ions was applied. Under this condition, the metal nuclei grow slowly and form uniform nanoparticles with high-adhesion strength on the porous silicon. ${ }^{9,20}$ The reactions between the metal and the porous silicon can be described as: ${ }^{21}$

$$
\begin{aligned}
& \text { Oxidation: } \mathrm{Si}+2 \mathrm{H}_{2} \mathrm{O} \longrightarrow \mathrm{SiO}_{2}+4 \mathrm{H}^{+}+4 \mathrm{e}^{-}, \\
& \text {Reduction: } \mathrm{Me}^{\mathrm{z}+}+\mathrm{ze}^{-} \longrightarrow \mathrm{Me}
\end{aligned}
$$

The energy states of the metal ion overlap with the valence band of silicon, enabling reduction to occur in the dark via the valence
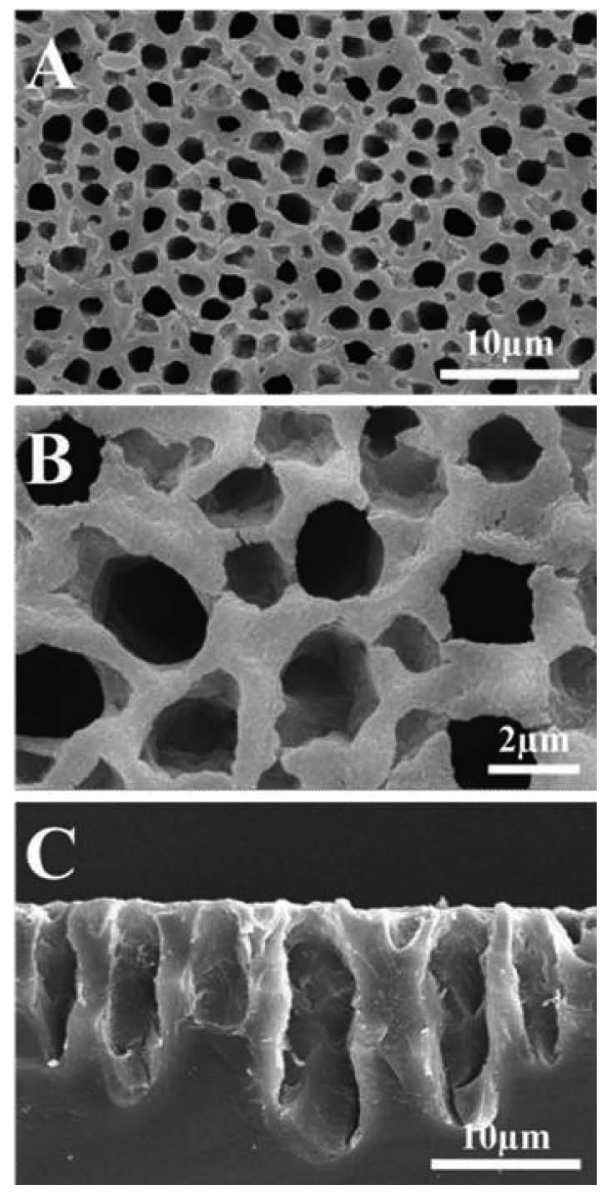

Fig. 2 FE-SEM images of porous silicon substrates at (A) low and (B) high magnifications. (C) Cross-section view of a porous silicon substrate.

band. ${ }^{20}$ This mechanism involves two reduction steps. The first step is silicon oxidation, in which holes from the metal ions can be injected into the valence band of porous silicon. In the next step, electron injection occurs from hydroxide $\left(\mathrm{Si}(\mathrm{OH})_{2}\right)$ into the conduction band of porous silicon. ${ }^{21}$ Hydrogen evolution takes place simultaneously. Compared with a flat silicon surface, metal nanoparticles can be more easily generated on the roughened silicon surface by the following process. The edge 

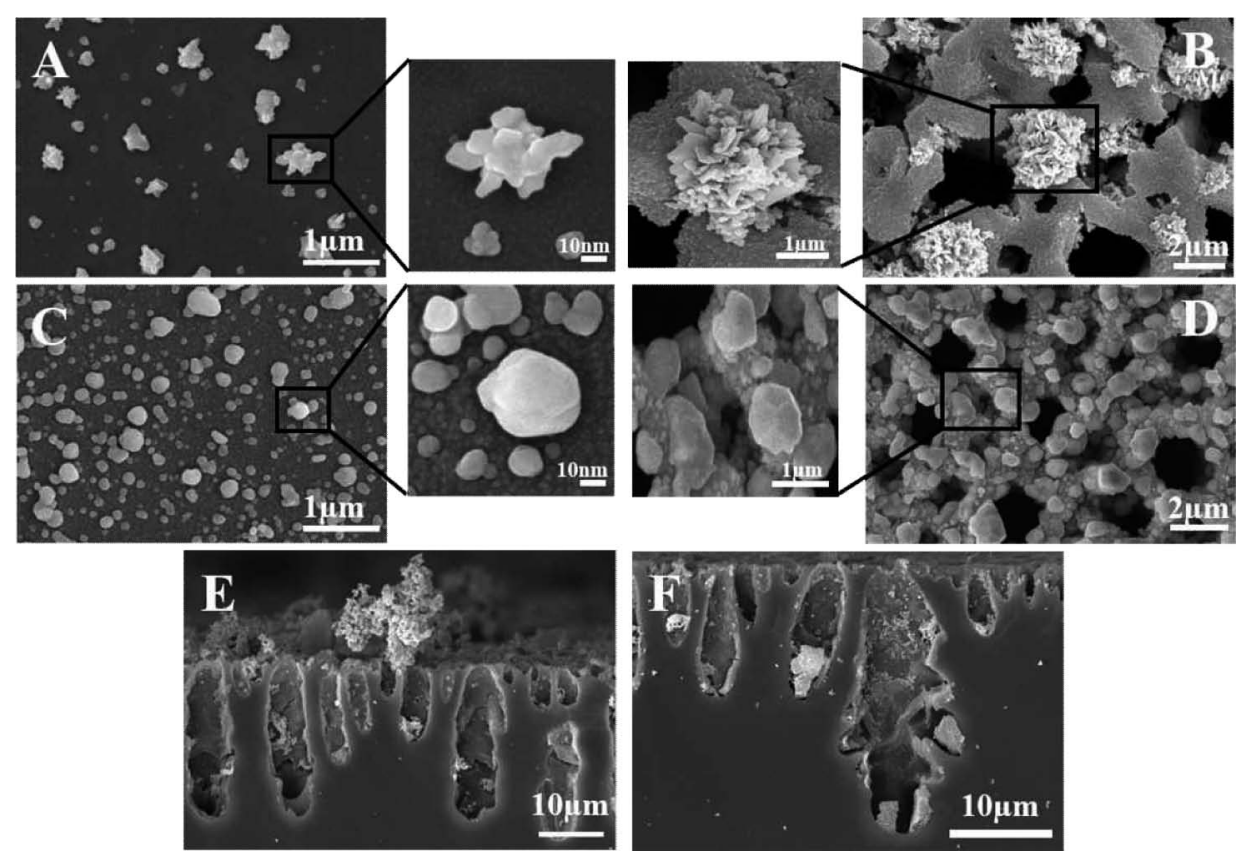

Fig. 3 FE-SEM images of (A) and (B) AuNPs on crystalline silicon and porous silicon, respectively; (C) and (D) AgNPs on crystalline silicon and porous silicon, respectively. FE-SEM images of (E) AuNPs and (F) AgNPs inside the pores of porous silicon, respectively.

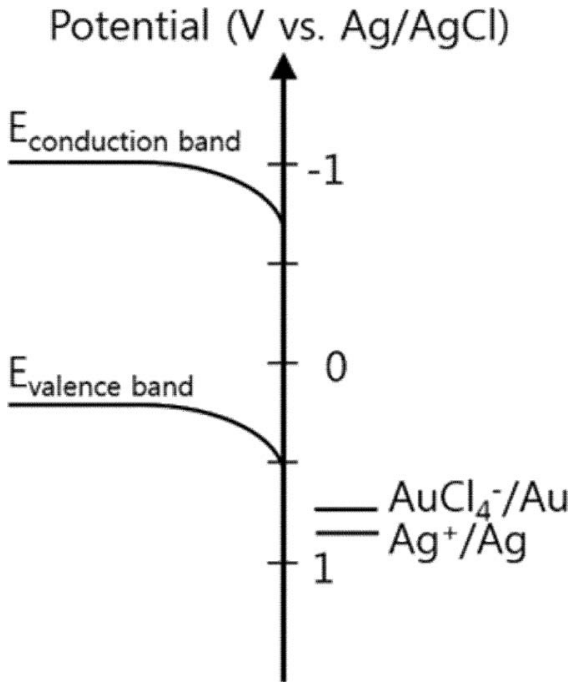

Fig. 4 Energy diagram for metal/metal ion complexes with respect to the position of the band edges for silicon surfaces in an aqueous solution at $\mathrm{pH} 2$.

of porous silicon induces a stronger electric field, resulting in a higher density of metal nanoparticles. In this case, the edges of the pore represent a unique type of surface defect, which possess areas of elevated reactivity and stimulate the reduction of metal ions. Additionally, the roughness of the surface provides a larger number of nucleation sites because the free energy for nucleation on the rough surface is lower than that on a flat surface. $^{22}$ Thus, a deposition of metal ions onto a porous silicon surface occurs at a higher rate than deposition onto conventional silicon wafers. ${ }^{23}$

The electrochemical detection of hydroxylamine by the
AuNPs-porous silicon sensor was performed in the absence and presence of $0.5 \mathrm{mM}$ hydroxylamine in $0.1 \mathrm{M}$ PBS ( $\mathrm{pH} 7.0$ ) by the cyclic voltammetry technique (Fig. 5(A)). Cyclic voltammograms of the AuNPs-porous silicon electrode show the electrocatalytic behavior of AuNPs-silicon electrodes toward hydroxylamine. No redox peaks were observed at the silicon electrodes without AuNPs (curves b and d). However, AuNPs-porous silicon electrodes exhibited high anodic peaks (curve a), which are attributed to the oxidation of hydroxylamine. These results showed that AuNPs effectively catalyze the oxidation process of hydroxylamine. Moreover, the anodic peak for AuNPs-porous silicon electrodes was higher than that for AuNPs-crystalline silicon electrodes (curve c), despite the fact that the oxidation potential of hydroxylamine on AuNPs-porous silicon electrodes is similar to that on AuNPs-crystalline silicon electrodes. This may be due to the difference in the active surface area of AuNPs on porous silicon and crystalline silicon. Figure 5(B) shows the calibration curve for the oxidation peak current of hydroxylamine on the AuNPs-porous silicon electrode. The current response had a linear correlation with the hydroxylamine concentration. The current changed linearly with concentrations ranging from $100 \mu \mathrm{M}$ to $1.32 \mathrm{mM}$ (linear regression coefficient, $R^{2}=0.995 ; n=3$ ) and the detection limit was determined to be as low as $55 \mu \mathrm{M}(S / N=3)$. The AuNPs-porous silicon electrode showed high sensitivity $\left(37.9 \pm 1.1 \mu \mathrm{A} \mathrm{mM}^{-1} \mathrm{~cm}^{-2}\right)$ in the linear range.

As another application, nanoparticle-decorated porous silicon substrates were used as substrates for SERS. SERS-based detection techniques are considered to be promising for chemical, biomedical, and environmental sensing systems because of their high sensitivity, wide applicability, little to no sample preparation requirements, and straightforward sample identification. $^{24}$ It is well-known that the SERS activities for certain nanostructured substrates largely depend on the activity and density of Raman hot spots. ${ }^{25}$ At the smallest interparticle spaces in the region of the separation, which are referred to as 

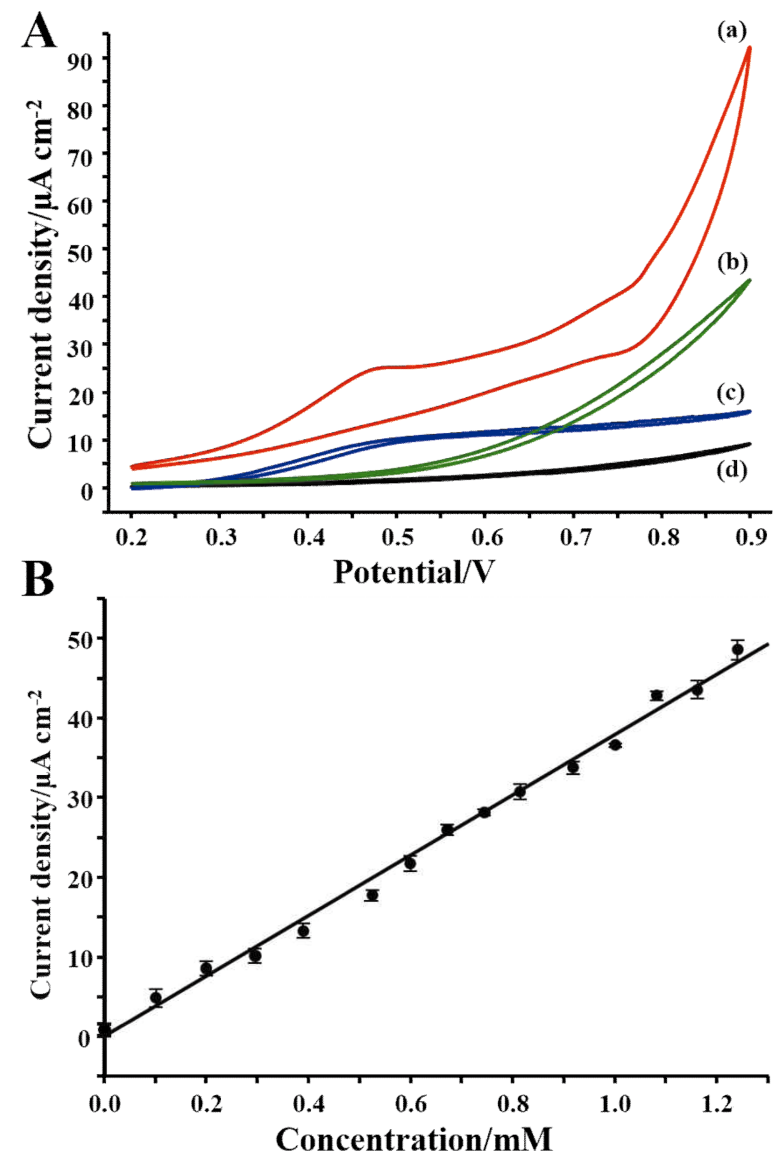

Fig. 5 (A) Cyclic voltammograms of $0.5 \mathrm{mM}$ hydroxylamine in $0.1 \mathrm{M}$ PBS (pH 7.0) on (a) AuNPs-porous silicon, (b) porous silicon, (c) AuNPs-crystalline silicon, and (d) crystalline silicon at a scan rate of $50 \mathrm{mV} / \mathrm{s}$. (B) Calibration curve for hydroxylamine detection on AuNPs-porous silicon $(n=3)$.

"hot spots", local electromagnetic field enhancement arises. ${ }^{26}$ Rough metallic electrodes play a major role as SERS substrates. The SERS activity was investigated by measuring the Raman signal of different concentrations of Rhodamine 6G dropped on the as-prepared silicon substrates. Figure 6(A) shows the comparisons of the SERS spectra on crystalline and porous silicon substrates. The SERS signal on the porous silicon substrates is stronger than on the crystalline silicon substrates. The morphology of the nanoparticles on the porous silicon substrates is rougher than that on the crystalline silicon substrates due to the fast nucleation and growth processes of nanoparticles caused by the strong electric field that is formed on the porous silicon substrates. Therefore, nanoparticles decorated on the porous silicon substrates have more Raman hot spots than those on the crystalline silicon substrates, resulting in a larger SERS signal. In addition, the AgNPs-porous silicon yielded higher signals compared to the AuNPs-porous silicon (Fig. 6(B)). The SERS signal enhancement seen with the silver particles was about five times higher compared to what was obtained with gold nanoparticles. This may be due to the interparticle Plasmon resonance enhancement of the closer-packed AgNPs (compared to AuNPs), as shown in Fig. 3. This is also in good agreement with the theoretical predictions that AgNPs exhibit much larger electromagnetic enhancement factors in the visible region (at least up to $\approx 600-650 \mathrm{~nm}$ ) than AuNPs. ${ }^{27}$ These results demonstrated that the AgNPs-porous
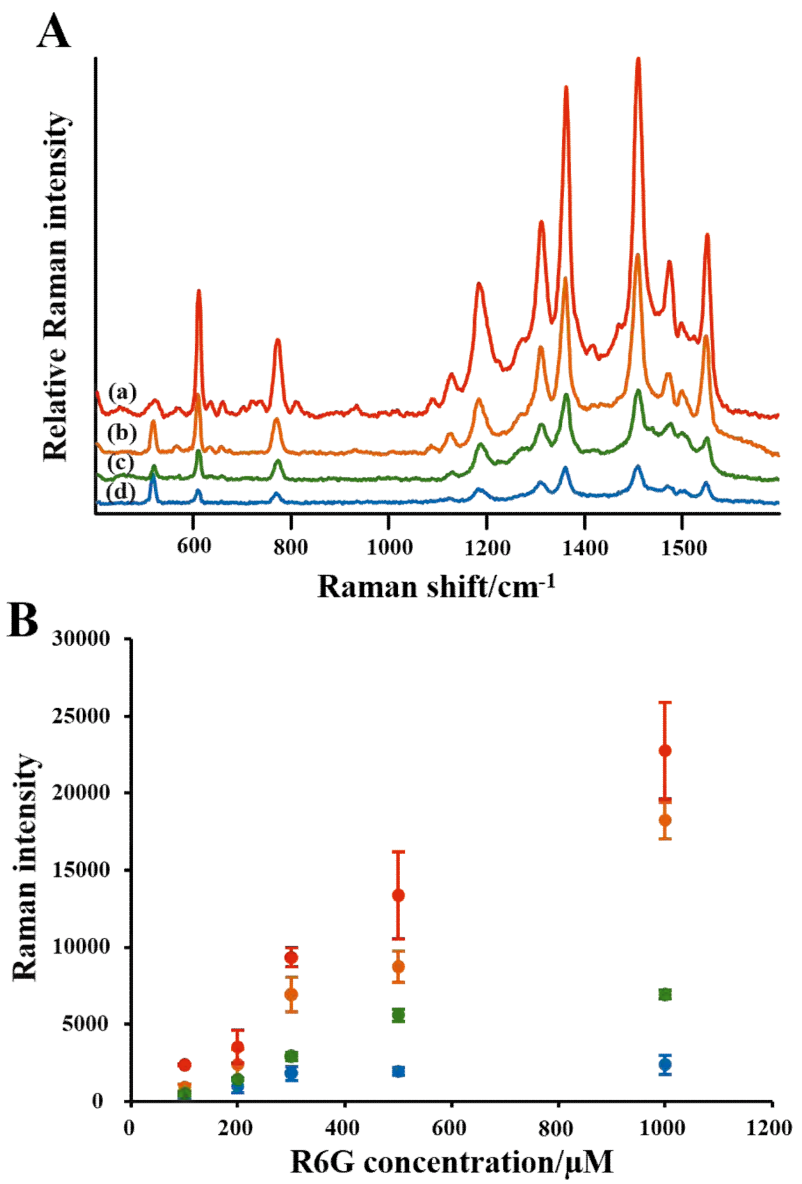

Fig. 6 (A) Surface-enhanced Raman spectra of Rhodamine 6G on (a) AgNPs-porous silicon, (b) AgNPs-crystalline silicon, (c) AuNPs-porous silicon, and (d) AuNPs-crystalline silicon. The concentration of Rhodamine $6 \mathrm{G}$ is $1 \mathrm{mM}$ and contains $1 \mathrm{mM} \mathrm{NaCl}$ in 1:1 solutions of water and ethanol. (B) Dependence of a Raman intensity on the Rhodamine $6 \mathrm{G}$ concentration.

silicon provides good SERS performance and relative uniformity across the substrate. We believe that the as-prepared substrate can provide reproducible SERS signals and possesses great potential for use in quantitative SERS analysis applications.

\section{Conclusions}

We fabricated nanoparticles on porous silicon using an electrochemical deposition method. These materials were then used as electrochemical sensing platforms for hydroxylamine detection and as SERS substrates. The formation of a porous structure was performed by electrochemical etching of n-type crystalline silicon in an aqueous HF solution with backside illumination. To stabilize the porous silicon surface, AuNPs and AgNPs were deposited onto the porous silicon electrochemically. A large number of uniform and stable nanoparticles can be decorated onto the porous structure to increase the electric-field strength. For hydroxylamine detection, the AuNPs-porous silicon electrodes showed good linearity for the concentration range from $100 \mu \mathrm{M}$ to $1.32 \mathrm{mM}$ with a sensitivity of $37.9 \mu \mathrm{A} \mathrm{mM}^{-1} \mathrm{~cm}^{-2}$ and a detection limit of $55 \mu \mathrm{M}$. As another application, the nanoparticle-decorated porous silicon substrates were used as substrates for SERS. The AgNPs-porous silicon substrate exhibited a SERS signal that was enhanced by a factor 
of five for Rhodamine $6 \mathrm{G}$ compared to the signal for AuNPs-porous silicon substrates. From these results, we believe that decorating nanoparticles onto porous silicon can be used to create promising analytical substrates for biochemical sensing in various fields.

\section{Acknowledgements}

This research was supported by the Basic Science Research Program through the National Research Foundation of Korea (NRF) funded by the Ministry of Science, ICT \& Future Planning (2008-0061891 and 2013R1A1A2054887).

\section{References}

1. J. D. Holmes, K. J. Ziegler, R. C. Doty, L. E. Pell, K. P. Johnston, and B. A. Korgel, J. Am. Chem. Soc., 2001, 123, 3743.

2. Y. Qu, L. Liao, Y. Li, H. Zhang, Y. Huang, and X. Duan, Nano Lett., 2009, 9, 4539.

3. D. Luo, L. Wu, and J. Zhi, ACS Nano, 2009, 3, 2121.

4. M. J. Sailor, "Porous Silicon in Practice", 2011, Chap. 1, Wiley-VCH Verlag GmbH \& Co. KGaA, Weinheim.

5. L. Gu, J.-H. Park, K. H. Duong, E. Ruoslahti, and M. J. Sailor, Small, 2010, 6, 2546.

6. F. Zhong and Z.-h. Jia, Phys. B, 2013, 411, 77.

7. C. K. Tsang, T. L. Kelly, M. J. Sailor, and Y. Y. Li, ACS Nano, 2012, 6, 10546.

8. J. Kanungo, S. Maji, A. K. Mandal, S. Sen, E. Bontempi, A. K. Balamurugan, A. K. Tyagi, K. Uvdal, S. Sinha, H. Saha, and S. Basu, Appl. Surf. Sci., 2010, 256, 4231.

9. M. P. Bui, S. Lee, K. N. Han, X. H. Pham, C. A. Li, J.
Choo, and G. H. Seong, Chem. Commun., 2009, 37, 5549.

10. G. G. Wildgoose, C. E. Banks, and R. G. Compton, Small, 2006, 2, 182.

11. H. Zhang and J. Zheng, Talanta, 2012, 93, 67.

12. M.-P. N. Bui, X.-H. Pham, K. N. Han, C. A. Li, E. K. Lee, H. J. Chang, and G. H. Seong, Electrochem. Commun., 2010, 12,250

13. E. C. Le Ru and P. G. Etchegoin, "Principles of SurfaceEnhanced Raman Spectroscopy", 2009, Chap. 1, Elsevier, Amsterdam.

14. A. Virga, P. Rivolo, F. Frascella, A. Angelini, E. Descrovi, F. Geobaldo, and F. Giorgis, J. Phys. Chem. C, 2013, 117, 20139.

15. H. Ko and V. V. Tsukruk, Small, 2008, 4, 1980.

16. V. Lehmann, J. Electrochem. Soc., 1990, 137, 653.

17. V. Lehmann and U. Gösele, Appl. Phys. Lett., 1991, 58, 856.

18. J. Jakubowicz, Superlattices Microstruct., 2007, 41, 205.

19. M. P. Stewart and J. M. Buriak, Adv. Mater., 2000, 12, 859.

20. G. Oskam, J. G. Long, A. Natarajan, and P. C. Searson, J. Phys. D: Appl. Phys., 1998, 31, 1927

21. M. Jeske, J. W. Schultze, M. Thönissen, and H. Münder, Thin Solid Films, 1995, 255, 63.

22. L.-C. Tien and Y.-J. Chen, Appl. Surf. Sci., 2012, 258, 3584.

23. F. A. Harraz, T. Tsuboi, J. Sasano, T. Sakka, and Y. H. Ogata, J. Electrochem. Soc., 2002, 149, C456.

24. X. Gong, Y. Bao, C. Qiu, and C. Jiang, Chem. Commun., 2012, 48, 7003.

25. E. C. Le Ru, M. Meyer, and P. G. Etchegoin, J. Phys. Chem. B, 2006, 110, 1944.

26. S. Akil-Jradi, S. Jradi, J. Plain, P.-M. Adam, J.-L. Bijeon, P. Royer, and R. Bachelot, RSC Adv., 2012, 2, 7837.

27. E. J. Zeman and G. C. Schatz, J. Phys. Chem., 1987, 91, 634. 